\title{
Rabaska
}

Revue d'ethnologie de l'Amérique française

PAPINEAU, SimON. Le Sens de l'humour absurde au Québec. Québec, Presses de l'Université Laval, "Quand la philosophie fait pop ! », 2012, 162 p. ISBN 978-2-7637-9869-1

\section{Sandrine Contant-Joannin}

Volume 14, 2016

URI : https://id.erudit.org/iderudit/1037481ar

DOI : https://doi.org/10.7202/1037481ar

Aller au sommaire du numéro

Éditeur(s)

Société québécoise d'ethnologie

ISSN

1703-7433 (imprimé)

1916-7350 (numérique)

Découvrir la revue

Citer ce compte rendu

Contant-Joannin, S. (2016). Compte rendu de [PAPINEAU, Simon. Le Sens de l'humour absurde au Québec. Québec, Presses de l’Université Laval, « Quand la philosophie fait pop ! », 2012, 162 p. ISBN 978-2-7637-9869-1]. Rabaska, 14,

263-266. https://doi.org/10.7202/1037481ar d'utilisation que vous pouvez consulter en ligne.

https://apropos.erudit.org/fr/usagers/politique-dutilisation/ 
qui veut comprendre le fonctionnement de ces appareils, les croquis sont très explicites. On peut y voir plusieurs modèles et leurs composantes, feu, bassin, pannes, cloisons, robinet, cheminée.

Dans la quatrième et dernière section de l'ouvrage, l'auteur présente un récit datant de 1876, dans lequel deux personnes partent courir les sucres à Metgermette (ancien nom de Saint-Zacharie et de Sainte-Aurélie). On comprend que l'époque, l'endroit et les personnages de l'histoire ne sont pas sortis de l'imaginaire de l'auteur, mais ont plutôt été inspirés par la tradition orale. Les protagonistes, en effet, sont Joseph Morin, 44 ans, du rang Saint-Nicolas, à Saint-Georges, et son fils Jérôme. On en déduit que l'auteur, lui-même un Morin, beauceron de surcroît, est en quelque sorte l'héritier de ce récit qui lui serait parvenu de bouche à oreille, de génération en génération.

Ce qui manque dans cet ouvrage, c'est l'interprétation de toute cette matière iconographique. L'auteur, dont on ne doute pas de l'expérience et du savoir, commente peu le volumineux corpus documentaire qu'il a pourtant rassemblé avec patience et longueur de temps. C'est d'autant plus dommage qu'on devine qu'il aurait beaucoup à dire. L'ouvrage n'en constitue pas moins une véritable petite mine d'or pour qui s'intéresse à l'évolution de l'instrumentation acéricole avant que la production de sirop d'érable ne connaisse les innovations technologiques de la fin du siècle dernier.

Bernard Genest

Ethnologue, Société québécoise d'ethnologie

Papineau, Simon. Le Sens de l'humour absurde au Québec. Québec, Presses de l'Université Laval, «Quand la philosophie fait pop! », 2012, 162 p. ISBN 978-2-7637-9869-1.

Publié en 2012, Le Sens de l'humour absurde au Québec est le mémoire de maîtrise de Simon Papineau (2006), actualisé pour sa publication aux Presses de l'Université Laval dans la collection « Quand la philosophie fait pop! » dirigée par Normand Baillargeon et Christian Boissinot. Titulaire d'une maîtrise en communication à l'Université du Québec à Montréal et d'un diplôme d'études supérieures spécialisées en design de jeux à l'Université de Montréal, Simon Papineau travaille dans le domaine des jeux vidéo. De 2011 à 2014, il a fait partie de l'Observatoire de l'humour, un groupe de recherche interdisciplinaire.

Observant la prolifération d'un humour qualifié « d'absurde » depuis quelques années, l'auteur a décidé d'approfondir le sujet. Il s'intéresse plus particulièrement au matériel comique qu'il dit moderne, soit à des textes, 
monologues, émissions et chansons produits après la défaite référendaire de 1996. Ses principaux objectifs sont de « définir, d'exemplifier, de catégoriser, de vulgariser et d'analyser " son objet de recherche (p. 2). Afin de mettre en lumière la profondeur d'un type d'humour que certains détracteurs pourraient qualifier de « vide », il combine approche psychosociale et analyse de contenu. L'auteur a fait appel à quelques ouvrages dont L'Ére du vide (1983), de Gilles Lipovetsky, a réalisé des entrevues avec des acteurs du milieu, et a fait de l'observation et de l'analyse de contenu humoristique. On le constate de par les méthodes de travail choisies, Papineau s'intéresse davantage au point de vue de l'émetteur, soit l'artiste, qu'à celui du récepteur.

D'entrée de jeu, Papineau affirme qu'il n'existe pas de définition scientifique officielle du style de comique auquel il s'intéresse, soulignant ainsi implicitement que cette thématique est peu développée. Afin de bien cerner l'objet de son étude, il se permet donc de le situer par rapport à de grands mouvements artistiques et il le définit comme : " un type d'humour qui se plaît à faire des associations entre des mots, des objets, des personnages, des lieux et des concepts qui n'ont, a priori, strictement aucun « rapport » [...] : la consécration de "l'anti-punch" » (p. 9). Il expose aussi divers éléments du contexte psychosocial actuel : la liberté, la tolérance, l'indifférence au sens, l'hédonisme, la dépolitisation et la psychologisation des individus, c'est-àdire le besoin d'exprimer ses émotions et de se questionner sur ses propres motivations et sentiments. Cela l'amène à envisager son objet d'étude comme un moyen d'exprimer les angoisses existentielles de l'individu, comme une manière de repousser les limites de la liberté et comme un mécanisme de défense social.

Papineau propose ensuite une classification personnelle des différents types d'humour établie à partir du modèle de l'anthropologue Jean-Pierre Desaulniers. Celle-ci permet de dégager trois formes de comique absurde moderne : la cérébrale, qui permet à l'humoriste de construire un univers avec une logique qui lui est propre ; la psychoaffective, qui met l'accent sur l'exagération des émotions; et la sociale, qui, contrairement aux deux autres, implique un désir d'aller vers le spectateur. C'est sur ces catégories que repose la suite de son analyse.

Dans le premier bloc analytique, portant sur l'humour absurde cérébral, Papineau présente le parcours et l'œuvre de Bruno Blanchet, le père de ce style, des Denis Drolet, un duo d'humoristes et musiciens, et de JeanThomas Jobin. Tous utilisent notamment les non-rapports, les longueurs, l'hyperprécision, les fausses corrélations, les décrochages et le " jouer faux » pour faire rire. Même si les propos qu'ils tiennent peuvent parfois sembler méchants, le contenu qu'ils proposent demeure globalement «"naïf”, gentil 
et inoffensif » (p. 71). Pour l'auteur, les artistes appartenant à cette catégorie sont ceux qui vont le plus loin dans leur irrationalité.

Dans la deuxième section, portant sur l'humour absurde psychoaffectif, l'auteur s'intéresse aux Chick'n swell, trio d'humoristes s'étant illustré sur scène et à la télévision. Abordant des termes sérieux tels que l'amour ou la mort, ceux-ci s'adressent aux émotions de l'individu. Les sketchs qu'ils proposent reposent sur leur manière de communiquer, c'est-à-dire sur leur jeu d'acteur, de même que sur la notion de non-rapport, les longueurs, etc. Il les décrit comme les représentants d'une génération « d'adulescents ».

Suivent, dans la troisième partie, les présentations des humoristes Patrick Groulx et Louis-José Houde, de même que des émissions Phylactère Cola et 3600 secondes d'extase, appartenant tous à la catégorie humour absurde social. Ce sont ceux qui proposent les univers les plus accessibles. Pour ces créateurs, ce type de discours est un moyen intéressant d'avoir plus de liberté. D'un côté, puisque l'humain est confronté à un nombre presque infini de choix et que rares sont les situations qui l'étonnent, l'absurde permet de surprendre le public. D'un autre côté, beaucoup d'idées semblent acceptables lorsqu'elles sont présentées de façon absurde. Le quatrième bloc se veut un « pot-pourri » permettant à l'auteur de présenter André Sauvé et quelques musiciens qu'il considère comme inclassables.

Finalement, l'auteur répond à sa question initiale en expliquant ce que cherche à exprimer l'humour absurde moderne. Permettant de sortir de la réalité en s'inventant un nouvel univers avec des règles et une logique particulières, il peut être compris comme un besoin de fuir. Sans être politique, il s'agit d'une prise de position puisque c'est un moyen d'exprimer sa désillusion. Il est également, d'une certaine manière, un combat, car il permet à l'individu de contester le fonctionnement d'une société où tout est « sensé, programmé, calculé, ordonné et logique » (p. 140). Il s'agit à la fois d'une façon d'exprimer les angoisses de l'individu, une manière d'affirmer un besoin de liberté absolue et un mécanisme de défense social. Les hypothèses émises par l'auteur sont ainsi confirmées.

Invitant le lecteur à faire une intéressante incursion dans le monde de l'humour moderne québécois, le livre de Simon Papineau, Le Sens de l'humour absurde au Québec, est pertinent et accessible. L'auteur trouve une nouvelle façon de dévoiler des traits de la société québécoise ou, du moins, d'une génération, en allant au-delà du cliché de l'individualisme et du désengagement social. Dans la conclusion de son livre, Papineau souligne l'importance de poursuivre les recherches dans le domaine qu'il a étudié soutenant que l'humour est un " véritable véhicule de désirs inconscients " (p. 150). À la suite de notre lecture, nous ne pouvons qu'être d'accord avec lui 
et souhaiter que des chercheurs de diverses disciplines explorent prochainement cette riche thématique.

\section{SANDrine Contant-Johnnin}

Université Laval

PARENT, FrÉDÉRIC. Un Québec invisible, enquête ethnographique dans un village de la grande région de Québec. Préface de MARCEL FourNIER. Québec, Presses de l'Université Laval, 2015, 281 p. ISBN 978-2-76372508-6.

Existe-t-il un « mystère de Québec » ou ne serait-ce pas là une étiquette commode pour camoufler, voire nier, ce qui crève les yeux par son évidence même ? Il y a des mystères qui n'en sont que par complaisance dans son aveuglement volontaire. Frédéric Parent, dans Un Québec invisible, se targue, non sans raison, de faire la lumière sur ce mystère qui obsède davantage ceux qui disent le constater que ceux qui en sont les principaux intéressés. Ce Québec dont il est question, est-il devenu invisible pour avoir été plus « tranquille» que ne le laissa croire sa révolution des années soixante du siècle dernier? Le préjugé urbain n'érige-t-il pas la campagne en conservatoire des valeurs d'un Québec dont il entend tourner la page ? Voilà une piste de réflexion qui n'est pas à négliger.

Dans cet essai fouillé, considéré par son préfacier, Marcel Fournier, " comme la meilleure recherche ethnographique récente en sociologie au Québec » (p. XII), l'auteur nous livre les résultats d'une enquête sur le terrain qu'il a menée en 2007-2008 dans une municipalité du Centre-du-Québec rebaptisée pour des considérations juridiques Sainte-Hélène-de-Lancaster ${ }^{4}$.

Le sous-titre de l'ouvrage mentionne qu'il s'agit d'une enquête ethnographique. Pour éviter toute méprise, il est impérieux de mettre les mots en harmonie avec la vérité des choses ainsi que le conseillait Confucius. La lecture de la bibliographie convaincra le lecteur qu'il parcourt une étude sociologique et qu'il n'est aucunement question de l'ethnographie des ethnologues. Cette ethnographie sert des fins sociologiques, tant par son orientation intellectuelle que par les matériaux qu'elle compile qui sont

4. Un esprit aiguisé aura tôt fait de découvrir l'identité de ce petit village de province. Marc Vallières, cité en bibliographie, a publié un article dans le Dictionnaire biographique du Canada 18911900, sur James King rebaptisé Queen par Parent. On saisit l'astuce, car il n'existe pas de James Queen dans le Dictionnaire biographique. En consultant l'article en question, on relève les informations mentionnées en page 33 d'Un Québec invisible. Il devient alors évident que Lancaster (Sainte-Anastasie-deNelson) est le pseudonyme de Lyster, appartenant à la MRC de l'Érable et non de l'Étoile comme il est écrit. On appréciera le procédé : la rime (Lancaster/Lyster), l'assonance (Étoile/Érable) et l'opposition (Queen/King). 